Recepción: 20 / 04 / 2017

Aceptación: 20 / 05 / 2017

Publicación: 15 / 07 / 2017
Ciencias de la Educación

Artículo Científico

\title{
Solucionario didáctico en el proceso de enseñanza aprendizaje de la estequiometria de las disoluciones
}

Didactic solution in the learning process of stoichiometry of solutions

\author{
Ensino Solucionario no processo de \\ aprendizagem da estequiometria de soluções
}

Freddy A. Pereira-Guanuche ${ }^{\text {I }}$ fpereira@utmachala.edu.ec

Kennya S. Ruiz-Veintimilla II kruiz@utmachala.edu.ec

Kennya M. Pereira-Ruiz ${ }^{\text {III }}$ kpereira@utmachala.edu.ec

Jorge M. Víllacis-Mosquera IV jvillacis@utmachala.edu.ec

Correspondencia: fpereira@utmachala.edu.ec 


\section{Resumen}

La química va más allá de las formulas y reacciones. Muchas de las cosas que ocurren a nuestro alrededor, tienen relación con ella. Recordemos que la química es una ciencia central y la estequiometria interviene en algún aspecto del mundo material, por lo que habrá siempre un interés, por el carácter fundamental de los materiales en cuestión. En el contenido se consideran los conceptos básicos que el estudiante debe saber sobre: Ecuaciones químicas (portadoras de información) acerca de reactivos y productos, Clases de reacciones químicas, Métodos de igualación de Ecuaciones químicas; estequiometria (medida) medidas y unidades, interpretación de ecuaciones estequiométricas, razón molar, relaciones entre moles, relaciones entre moles, relaciones entre moles y gramos, relaciones entre gramos, relaciones gramos a moles, reactivo limitante, reactivo en exceso, rendimiento teórico y rendimiento real, rendimiento y pureza; soluciones; soluciones diluidas y soluciones concentradas, formas de expresar concentración, unidades físicas de concentración, unidades químicas de concentración, pesos equivalentes, normalidad, fracción molar, molalidad, disoluciones y titulación. En el sistema de ejercicios se consideró problemas comunes del medio, en que se aplicaron para su resolución términos y formulas practicadas en el primer módulo del diplomado que servirán para aprobar el conocimiento y desarrollar la habilidad del estudiante

Palabras clave: Química; ciencia; estequimetría; ecuaciones; estudiantes; ejercicios. 


\section{Abstract}

Chemistry goes beyond formulas and reactions. Many of the things that happen around us are related to it. Recall that chemistry is a central science and stoichiometry intervenes in some aspect of the material world, so there will always be an interest, because of the fundamental nature of the materials in question. In the content are considered the basic concepts that the student should know about: Chemical equations (information carriers) about reagents and products, Classes of chemical reactions, Equation methods of Chemical equations; Stoichiometric equations, molar ratios, mole ratios, mole ratios, mole-grams ratios, grams ratios, grams-to-moles ratios, limiting reactants, excess reagents, theoretical yields and yields Real, yield and purity; Solutions; Diluted solutions and concentrated solutions, concentration forms, physical units of concentration, chemical units of concentration, equivalent weights, normality, molar fraction, molality, dissolution and titration. In the system of exercises considered common problems of the environment, in which were applied for their resolution terms and formulas practiced in the first module of the diploma that will serve to approve the knowledge and develop the student's ability

Keywords: Chemistry; science; stethoscope; equations; students; exercises. 


\section{Resumo}

Química ultrapassa as fórmulas e reacções. Muitas das coisas que acontecem ao nosso redor, estão relacionados a ela. Lembre-se que a química é uma ciência central e estequiometria envolvido em algum aspecto do mundo material, de modo que sempre haverá um interesse, a natureza fundamental dos materiais envolvidos. Conteúdo são considerados os conceitos básicos que o estudante deve saber sobre: equações químicas (portadores de informação) em reagentes e produtos, aulas de reações químicas, métodos de equalização equações químicas; estequiometria (medidas) medições e unidades de interpretação, de equações estequiométricas, relação molar, as relações entre moles, relações de razões molares entre moles e em gramas, as relações entre gramas, relações gramas a moles, de reagente limitante, reagente excessiva, rendimento teórico e desempenho verdadeiro, rendimento e pureza; soluções; e soluções diluídas concentrados, maneiras de expressar a concentração, as unidades de concentração físicas, as unidades de concentração química, pesos equivalentes, fracção normal, em moles, molalidade, as soluções e as soluções de titulação. No sistema de exercício foram consideradas problemas ambientais comuns, eles foram aplicados para prazos de liquidação e fórmulas feitas no primeiro módulo do diploma que irá servir para passar o conhecimento e desenvolver a capacidade do aluno

Palavras-chave: Química; ciência; estequiometria; equações; estudantes; exercícios. 


\section{Introducción}

Luego de analizar los diferentes tipos de reacciones se puede estudiar los aspectos cuantitativos. Las reacciones ponderales (o de Masa) entre reactivos y productos, representan la ESTEQUIOMETRIA $($ griego Stoicheion $=$ Elemento y Metron $=$ Medida $)$ de la relación .

Para su interpretación cuantitativa se requiere aplicar el conocimiento de las Masas Morales y el concepto del Mol. En muchos cálculos estequiométricos se pregunta "si se conocen las cantidades de las sustancias iniciales (esto es, los reactivos) en una reacción”, ¿Qué cantidades de producto se formara?

Tratamos en el siguiente trabajo desarrollar, un solucionario de ejercicios de aplicación de las leyes estequiométricas en los cálculos químicos en sustancias en Disolución dada la necesidad de explicar el desarrollo de los procesos químicos a partir de la estructura de las sustancias y las leyes que los gobiernan, observando su uso correcto y el equilibrio del medio ambiente.

Se pretende organizar conceptos y resolver problemas de la estequiometria de las disoluciones para mejorar la formación integral de los alumnos del primer año de la Facultad de Ciencias Químicas.

Las ecuaciones balanceadas son la base para hacer cálculos y resolver ejercicios numéricos acerca de cantidades de productos que puedan obtenerse a partir de una cantidad dada de reactivos, cantidad de un reactivo que se combina con una cantidad dada de otro, o de la cantidad de un reactivo que se requiere para obtener cierta cantidad de un producto. Cada símbolo y cada formula de una ecuación significa una cantidad específica de elementos y compuestos que permiten interpretar cuantitativamente las ecuaciones en términos de números de moles y números de gramos. Cuando en las reacciones químicas están involucrados gases los cálculos estequiométricos implican también relaciones de volúmenes. (Cárdenas F. Gelves C. 1998) 


\section{Materiales y métodos (Metodología).}

En el contenido se considera los conceptos básicos que el estudiante debe saber sobre: Ecuaciones químicas (portadoras de información) acerca de reactivos y productos, Clases de reacciones químicas, Métodos de igualación de Ecuaciones químicas, estequiometria (medida) medidas y unidades, interpretación de ecuaciones estequiométricas, razón molar, relaciones entre moles, relaciones entre moles, relaciones entre moles y gramos, relaciones entre gramos, relaciones gramos a moles, reactivo limitante, reactivo en exceso, rendimiento teórico y rendimiento real, rendimiento y pureza; soluciones; soluciones diluidas y soluciones concentradas, formas de expresar concentración, unidades físicas de concentración, unidades químicas de concentración, pesos equivalentes, normalidad, fracción molar, disoluciones y titulación.

En el solucionario de ejercicios se consideró problemas comunes del medio, en que se aplicaron para su resolución términos y formulas.

\section{Resultados.}

\section{Conversión de Unidades (Equivalentes)}

\section{MASA}

1 Slug $=14.6 \mathrm{~kg}$ $1 \mathrm{Kg}=0.0685 \mathrm{slug}$

$1 \mathrm{~kg}$ tiene un peso de $2.20 \mathrm{lb}$ en lugares donde $\mathrm{g}=9.81 \mathrm{~m} / \mathrm{s}^{2} \mathrm{~J}$

1libra (Lb) (sistema anglosajón)

1 tonelada (ton), (menor o americana)

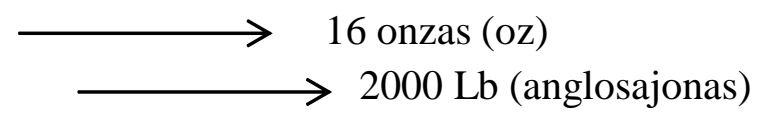

\section{FUERZA}
$\mathbf{1} \mathbf{L b}=4.45 \mathrm{~N}$
$\mathbf{1 N}=\operatorname{dina}=0.225 \mathrm{Lb}$

\section{PRESIÓN}

$1 \mathrm{~atm}=1.013 \mathrm{bar}=1.013 \times \mathrm{N} / \mathrm{m}^{2}=14.7 \mathrm{Lb} / \mathrm{pulg}^{2}=760$ torr 
$1 \mathrm{Lb} / \mathbf{p u l g}^{2}=6.90 \times 10^{3} \mathrm{~N} / \mathrm{m}$

$1 P a=1 \mathrm{~N} / \mathrm{m}^{2}=1.45 \times \mathrm{Lb} / \mathrm{pulg}^{2}$

$1 \mathbf{L b} / \mathbf{p u l g}^{2}(\mathbf{P S I})=2.036$ pulg de $\mathrm{Hg}$ a $0{ }^{\circ} \mathrm{C}$

$1 \mathbf{L b} /$ pulg$^{2}(\mathbf{P S I})=2.311$ pies de agua a $70^{\circ} \mathrm{F}$

\section{DENSIDAD}

$1 \mathrm{~mol}-$ gr de un gas ideal a $0{ }^{\circ} \mathrm{C}, 760 \mathrm{mmHg}=22.4140$ litros $=22.4140 \mathrm{~cm}^{3}$

$1 \mathrm{~mol}-$ gr de un gas ideal a $0{ }^{\circ} \mathrm{C}, 760 \mathrm{mmHg}=359.05$ pies cúbicos

Densidad del aire seco a $0{ }^{\circ} \mathrm{C}, 760 \mathrm{mmHg}=1.2929$ gramos $\mathrm{X}$ litro $=0.080711 \mathrm{Lb} X$ pie $^{3}$

1 gramo por $\mathrm{cm}^{3}=62.43 \mathrm{Lb} \mathrm{X}$ pie $^{3}$

1 gramo por $\mathrm{cm}^{3}=8.345$ Lb X galón U.S.A.

\section{ESCALAS DE TEMPERATURA}
${ }^{\circ} \mathbf{F}=1.8^{\circ} \mathrm{C}+32$
${ }^{\circ} \mathbf{K}={ }^{\circ} \mathrm{C}+273.16$
${ }^{\circ} \mathbf{R}={ }^{\circ} \mathrm{F}+459.69$

\section{VOLUMEN}

1 pulgada cubica $=16.39 \mathrm{~cm}^{3}$

1 galón U.S.A. $($ americano) $=37.85$ litros

\section{OTROS DATOS UTILES}

Equivalentes en Joules $(1 \mathrm{cal})--$

Cero Absoluto $\left({ }^{\circ} \mathrm{k}\right)$--- $273.15^{\circ} \mathrm{C}$

Tierra: Masa---------------------------------------------------- 5.97 x Kg

Luna: Masa $7.35 \times \mathrm{Kg}$

Sol: Masa ------------------------------ 1.99 x Kg

\section{CONSTANTES FUNDAMENTALES}

\begin{tabular}{|c|c|c|c|}
\hline CANTIDAD & SIMBOLO & VALOR APROXIMADO & $\begin{array}{c}\text { MEJOR VALOR } \\
\text { ACTUAL }\end{array}$ \\
\hline Numero de Abogador & NA & $6.02 \mathrm{X}$ & $6.0221367(36)$ \\
\hline
\end{tabular}


Freddy A. Pereira-Guanuche; Kennya S. Ruiz-Veintimilla; Kennya M. Pereira-Ruiz; Jorge M. Víllacis-Mosquera

\begin{tabular}{|c|c|c|c|}
\hline $\begin{array}{c}\text { Constante de los } \\
\text { gases }\end{array}$ & $\mathrm{R}$ & $\begin{array}{c}8.315 \mathrm{~J} / \mathrm{mol} \\
\mathrm{K}=1.99 \mathrm{cal} / \mathrm{mol}{ }^{\circ} \mathrm{K} \\
=0.082 \mathrm{~atm} \times \text { litro } / \mathrm{mol}^{\circ} \mathrm{K}\end{array}$ & $8.314510 \mathrm{~J} / \mathrm{mol}^{\circ} \mathrm{K}$ \\
\hline $\begin{array}{c}\text { Masa en reposo del } \\
\text { electrón }\end{array}$ & & 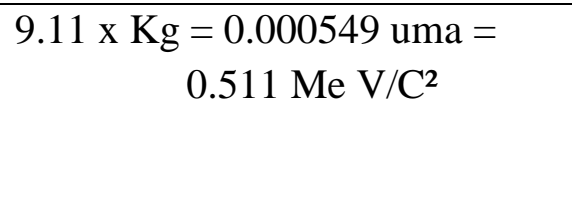 & $\begin{array}{c}9.1093897(54) \times \\
\mathrm{Kg} \\
548579903(13) \\
\text { uma }\end{array}$ \\
\hline $\begin{array}{c}\text { Masa en reposo del } \\
\text { protón }\end{array}$ & & $\begin{array}{c}1.6726 \times \mathrm{Kg}=1.00728 \mathrm{uma} \\
=938.3 \mathrm{Me} \mathrm{V} / \mathrm{C}^{2}\end{array}$ & $\begin{array}{c}1.6749286(10) \mathrm{x} \\
\mathrm{Kg} \\
1.007276470(12) \mathrm{uma}\end{array}$ \\
\hline $\begin{array}{c}\text { Masa en reposo del } \\
\text { neutrón }\end{array}$ & & $\begin{array}{c}1.6749 \times \mathrm{Kg}=1.008665 \mathrm{uma} \\
=939.6 \mathrm{Me} \mathrm{V} / \mathrm{C}^{2}\end{array}$ & $\begin{array}{c}1.6749286(10) \mathrm{x} \\
\mathrm{Kg} \\
1.008664904(14) \mathrm{uma}\end{array}$ \\
\hline $\begin{array}{l}\text { Unidad de masa } \\
\text { atómica }\end{array}$ & 1 uma & $\begin{array}{c}1.6605 \mathrm{Kg}=931.5 \mathrm{Me} \\
\mathrm{V} / \mathrm{C}^{2}\end{array}$ & $\begin{array}{c}1.6605402(10) \mathrm{x} \\
\mathrm{Kg} \\
931.494(28) \mathrm{Me} \mathrm{V} / \mathrm{C}^{2}\end{array}$ \\
\hline
\end{tabular}

\section{FÓRMULAS ESTEQUIOMETRICAS}

\section{MASAS ATÓMICAS}

UNIDADES DE MASA ATÓMICA = parte 12/6 C

$$
1 \mathrm{U}=\mathrm{m}(12 / 6) / 12 \quad 1 \mathrm{U}=16605402 \times \mathrm{Kg}
$$

MASA ATOMICA RELATIVA $=\operatorname{Ar}(\mathrm{X})$

$$
\text { ( ) }
$$

MASA FORMULA RELATIVA = $\operatorname{Mr}(X)$

$\operatorname{Mr}(\mathrm{O})=2 \operatorname{Ar}(\mathrm{H})+\operatorname{Ar}(0)=2 \times 1.008+15.9950=18.011 \mathrm{U}$

CANTIDAD DE SUSTANCIA = N Partículas (átomos, moléculas, iones, neutrones)

UNIDAD DE CANTIDAD DE SUSTANCIAS = 6.022167 X Avogadro $(\mathrm{N})$

MASA MOLECULAR = 6.022167 X átomos, moléculas, iones, neutrones, etc. 
$=\mathrm{M}(\mathrm{O})=18.0110 \mathrm{~g}$ (Es la masa de $6.022167 \mathrm{X}$ moléculas $)$

$$
=\operatorname{Mr}(\mathrm{O})=18.0110 \mathrm{U}(\text { Es la masa de una molecula })
$$

\section{RELACION ENTRE LA CANTIDAD DE SUSTANCIAS Y LA MASA}

$$
=\underline{()}
$$

$\mathbf{n}=$ Cantidad de sustancias,$\quad \mathbf{m}=$ Masa, $\mathbf{M}=$ Masa molar

$$
\text { ( ) } \quad \text { V }=\text { Volumen, } d=\text { Densidad }
$$

EQUIVALENTE QUIMICO $=\mathrm{X} / \mathrm{Z}^{*}$

$$
\begin{aligned}
\mathbf{X} & =\text { Sustancia compuesta por partículas. } \\
\mathbf{Z}^{*} & =\text { Numero de equivalencia } \\
& =\text { Numero de para un acido } \\
& =\text { Numero de para una base } \\
& =\text { Numero de cargas }(+) \text { o (-) para un oxido o una sal } \\
& =\text { Numero de e transferidos en un proceso Redox }
\end{aligned}
$$

CANTIDAD DE SUSTANCIA EN EQUIVALENTES = Número de fracciones

$\mathbf{N}\left(\mathbf{X} / \mathbf{Z}^{*}\right)=\mathrm{Z}^{*} \mathrm{n}(\mathrm{x})$

MASA MOLAR EN EQUIVALENTES = Es la masa de un mol de fracciones X/Z* de la partícula $\mathrm{X}$.

M (

$$
)=\stackrel{()}{-}=49.0 \mathrm{gr}
$$

\section{RELACIONES QUE DERIVAN DE LAS LEYES ESTEQUIOMETRICAS EN FÓRMULAS QUÍMICAS}

\section{Aa $\mathbf{B b}$}




$$
\begin{aligned}
& \frac{(\mathrm{)})}{(\mathrm{)}}=\frac{(\mathrm{)}}{(\mathrm{)}} \\
& \frac{()}{()}=\frac{()}{()} \\
& \tau_{()}^{()}= \\
& \frac{()}{(\quad)}= \\
& \frac{(*)}{(*)}=1 \\
& \frac{(*)}{(*)}=1
\end{aligned}
$$

RELACIONES ENTRE MASAS REACTIVO LIMITANTE EN EXCESO

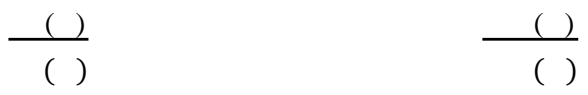

RELACIONES ENTRE CANTIDADES DE SUSTANCIAS

$$
\frac{(\text { ) }}{(~)}
$$

RELACIONES ENTRE CANTIDADES DE SUSTANCIA EN EQUIVALENTE

$\begin{array}{cc}\frac{(*)}{(*)} & \text { Rct }=1 \\ \frac{(*)}{(*)} & \text { Rct }=1\end{array}$

DISOLUCIONES

CONCENTRACION DE LAS DISOLUCIONES

RELACIONES ENTRE MASAS, VOLUMENES, CANTIDADES DE SUSTANCIA Y CANTIDADES DE SUSTANCIA EN EQUIVALENTES

$\mathbf{X}=$ Soluto $\quad \mathbf{d i}=$ Disolvente $\quad \mathbf{D}=$ Disolución $\quad \mathbf{d}=$ Densidad

QUE RELACIONAN MASAS: Fracción Másica (W(X))

$\mathbf{W}(\mathbf{X})=\frac{(\mathrm{)})}{(\mathrm{)}}$ Adimensional FRACCION MASICO =

PUEDE EXPRESARSE EN: \%, por mil, por millón (ppm) de partes de disolución 
$\mathbf{W}(\mathbf{X})=()$

\section{QUE RELACIONAN MASAS: RELACION MASICA}

Se emplea para expresar El coeficiente de solubilidad $(\mathbf{S}(\mathbf{X}))$

$\mathbf{S}(\mathrm{X})=\frac{()}{()} \quad$ Coeficiente De Solubilidad $=$

(Generalmente en \%)

Determine que masa de $\mathbf{O}$ está contenida en una muestra de 220.0 de

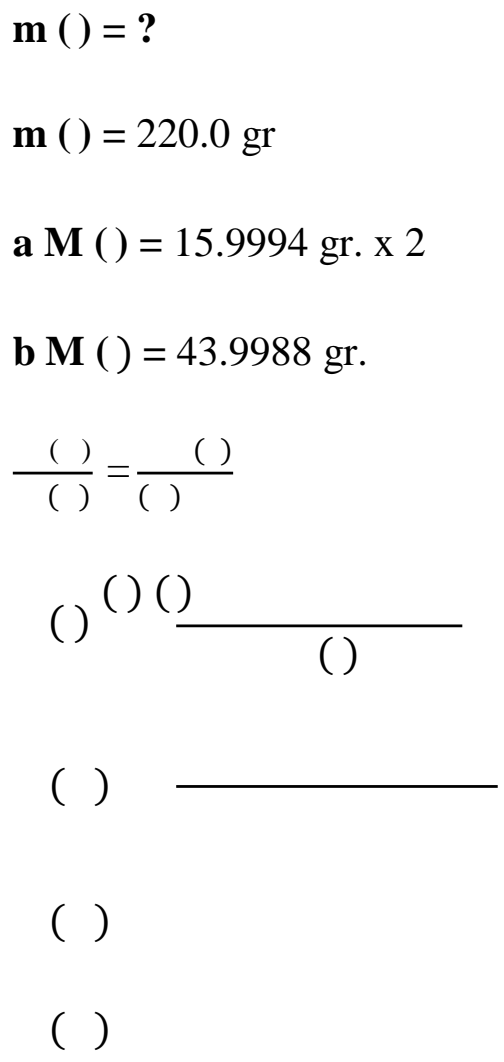

\section{REACCION QUIMICA}

Proceso en el cual una sustancia (o sustancias) cambia para formar una o mas sustancias nuevas.

Las reacciones químicas se representan mediante ecuaciones químicas.

Por ejemplo: 


$$
\begin{gathered}
2 \mathrm{CO}(\mathrm{g})+\quad(\mathrm{g}) \rightarrow 2 \mathrm{C} \quad(\mathrm{g}) \\
2 \mathrm{HgO}(\mathrm{g}) \rightarrow \quad(\mathrm{g})+\quad(\mathrm{g})
\end{gathered}
$$

Para describir lo que sucede cuando se agrega cloruro de sodio $(\mathrm{NaCl})$ al agua, se escribe:

$$
\mathrm{NaCl}(\mathrm{s}) \rightarrow \mathrm{NaCl}(\mathrm{ac})
$$

Donde ac significa disolución acuosa. Al escribir sobre la flecha se indica el proceso físico de disolver una sustancia en agua, aunque algunas veces no se pone, para simplificar.

El conocimiento del estado físico de los reactivos y productos es muy útil en el laboratorio, por ejemplo, cuando reaccionan el bromuro de potasio $(\mathrm{KBr})$ y el nitrato de plata $(\mathrm{AgN})$ en medio acuoso se forma un sólido, el bromuro de plata (ArBr).

$$
\mathrm{KBr}(\mathrm{ac})+\mathrm{AgN}(\mathrm{ac}) \rightarrow \mathrm{KN}(\mathrm{ac})+\mathrm{AgBr}(\mathrm{s})
$$

Si no se indican los estados físicos de los reactivos y productos, una persona no informada podría tratar de realizar la reacción al mezclar $\mathrm{KBr}$ solido con $\mathrm{AgN}$ solido, que reaccionan muy lentamente o no reaccionan.

\section{REACCIONES SECUENCIALES}

A menudo se necesita más de una reacción para cambiar los materiales de partida en los productos deseados. Esto se comprueba en muchas reacciones que realizamos en el laboratorio y en muchos procesos industriales. Se llaman REACCIONES SECUENCIALES. La cantidad de producto deseado de cada reacción se toma como material de partida para la siguiente.

\section{EJEMPLO:}

A altas temperaturas el carbono reacciona con agua para producir una mezcla de $\mathrm{CO}$ e $\mathrm{H}$.

$$
\mathrm{C}+\mathrm{O} \rightarrow \mathrm{CO}
$$

El monóxido de carbono se separa del y se usa para separar el níquel del cobalto formando un compuesto volátil, tetracarboniquel $\mathrm{Ni}(\mathrm{C})$.

$$
\mathrm{Ni}+4 \mathrm{CO} \rightarrow \mathrm{Ni}(\mathrm{C})
$$


¿Qué masa de ni(c) . Podria obtenerse del co producido por la reaccion de 75.0 de carbono? Suponer el $100 \%$ de reacción y el $100 \%$ de recuperación en las 2 etapas?

\section{PLANTEAMIENTO}

Interpretando ambas ecuaciones químicas en la forma habitual y resolviendo el problema en 2 etapas. Veremos que un mol de $\mathrm{C}$ produce un mol de $\mathrm{CO}$ y que se necesita 4 moles de $\mathrm{CO}$ para producir un mol $\mathrm{Ni}(\mathrm{C})$.

1) Determinamos el \# de moles de CO formados en la primera reacción

2) Del número de moles de $\mathrm{CO}$ producidos en la primera reacción calculamos el número de gramos de $\mathrm{Ni}(\mathrm{C})$ que podría formarse en la segunda reacción.

$\begin{array}{lll}\mathrm{C}+\mathrm{O} & \rightarrow & \mathrm{CO}+ \\ 1 \mathrm{~mol} 1 \mathrm{~mol} & & 1 \mathrm{~mol} 1 \mathrm{~mol}\end{array}$

$\mathrm{X}$ moles de $\mathrm{CO}=75.0$ gr $\mathrm{x} X=6.25$ moles $\mathrm{CO}$

$$
\mathrm{Ni}+4 \mathrm{CO} \quad \rightarrow \quad \mathrm{Ni}(\mathrm{C})
$$

$\mathrm{X}$ gr Ni $(\mathrm{C})=6.25$ moles $\mathrm{CO}$

$\mathrm{x}^{()} \mathrm{X}^{()}=267 \operatorname{gr~N}()$

Alternativamente podemos establecer una serie de factores de unidad basados en las conversiones de la secuencia de reacción y resolver el problema de una vez.

$\mathrm{X} \operatorname{gr~Ni}(\mathrm{C})=$

$75 \mathrm{Cx} \times()-\longrightarrow \frac{()}{()}=267 \operatorname{gr} \mathrm{g}()$

\section{REACTIVOS LIMITANTES}

En una reacción química, una vez haya consumido uno de los reactivos la reacción se para.

$$
2() \quad(\text { ) }
$$


Así si queremos obtener agua a partir de 10 moles de hidrogeno y 7 moles de oxígeno, como la estequiometria de la reacción es 2 moles de hidrogeno reacciona con un mol de oxígeno para dar dos moles de agua, una vez haya reaccionado todo el hidrogeno nos quedaran dos moles de y se habrá obtenido 10 moles de agua.

El reactivo que se consumido en su totalidad en una reacción química se le denomina reactivo limitante, ya que limita la cantidad de producto formado. Así en el ejemplo anterior el hidrogeno era el reactivo limitante, ya que con los 7 moles de oxigeno podríamos haber obtenido 14 moles de agua.

\section{¿Cómo operar para conocer cuál es el reactivo limitante de una reacción?}

Calculamos los moles de producto que se obtienen con cada reactivo, suponiendo que el resto reactivos están en cantidad suficiente. Aquel reactivo que nos del menor número potencial de moles de producto es el reactivo limitante. Al resto de reactivos, presentes en mayor cantidad que la necesaria para reaccionar con la cantidad del reactivo limitante, se les denomina reactivos en exceso.

Considere la siguiente reacción:

$$
2 \mathrm{~N}(\mathrm{~g})+\mathrm{C}(\mathrm{g}) \rightarrow(\mathrm{N}) \mathrm{CO}(\mathrm{ac})+(\mathrm{)}
$$

Suponga que se mezclan 637,2 g de con 1142 g de ¿Cuántos gramos de urea [(N )

$\mathrm{CO}]$ se obtendrán?

1. Primero tendremos que convertir los gramos de reactivos en moles:

637,2 gramos de () $\mathrm{x} \quad \longrightarrow=37,42$ moles ( )

$1142 \operatorname{gramos}$ de ( ) $\mathrm{x} \quad-=25,95$ moles ()

2. Ahora definimos la proporción estequiométrica entre reactivos y productos:

A partir de 2 moles de se obtiene $1 \mathrm{~mol}$ de $(\mathrm{N}) \mathrm{CO}$ 
A partir de 2 moles de se obtiene $1 \mathrm{~mol}$ de $(\mathrm{N}) \mathrm{CO}$

3.-Calculemos el número de moles de producto que se obtendrían si cada reactivo se consumiese en su totalidad:

37,2 moles de ()$\times\left({ }^{(}\right)=18.71$ moles $(\mathrm{N}) \mathrm{CO}$

25,95 moles de ()$\left.\times{ }^{(}\right)=25.95$ moles $(\mathrm{N}) 2 \mathrm{CO}$

()

1. El reactivo limitante es el ( ) y podremos obtener como máximo 18.71 moles de urea.

2. Y ahora hacemos la conversión a gramos:

$18.71 \mathrm{molde}(\mathrm{N}) \quad \mathrm{CO} \mathrm{x}^{\frac{()}{}}=1124(\mathrm{~N}) \mathrm{CO}$

\section{Ejercicio 1,-}

La aspirina se produce a partir del ácido salicílico y el anhídrido acético

a) ¿Cuánto acido salicílico se requiere para producir 1,5x $\mathrm{kg}$ de aspirina, suponiendo que todo el ácido salicílico se convirtiera en aspirina?

b) ¿Cuánto ácido salicílico se requerirá si solo el $80 \%$ del ácido salicílico se convierte en aspirina?

c) ¿Cuál es el rendimiento teórico de aspirina si reacciona $185 \mathrm{~kg}$ de ácido salicílico con $125 \mathrm{~kg}$ de anhídrido acético?

d) Si la situación descrita en la parte (c) produce $182 \mathrm{~kg}$ de aspirina, ¿Cuál es el porcentaje de rendimiento?

\section{$\underline{\text { Datos }}$}

$\mathrm{M}($ Ácido salicílico $)=183 \mathrm{~g} / \mathrm{mol}($ masa molar $)$

Anhídrido acético = $102 \mathrm{~g} / \mathrm{mol}$ (masa molar) 
Aspirina $=180 \mathrm{~g} / \mathrm{mol}($ masa molar $)$

a)

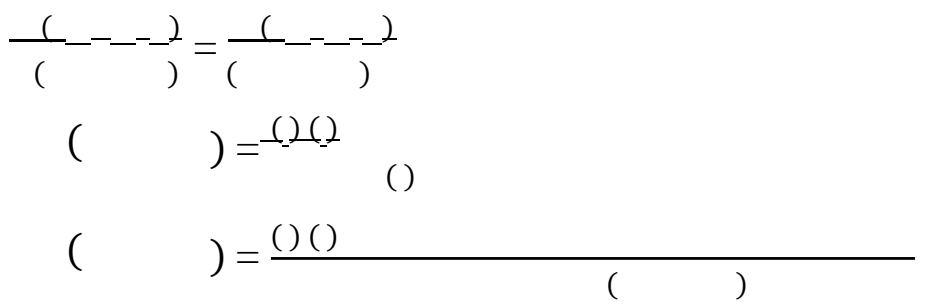

()$=$

b)

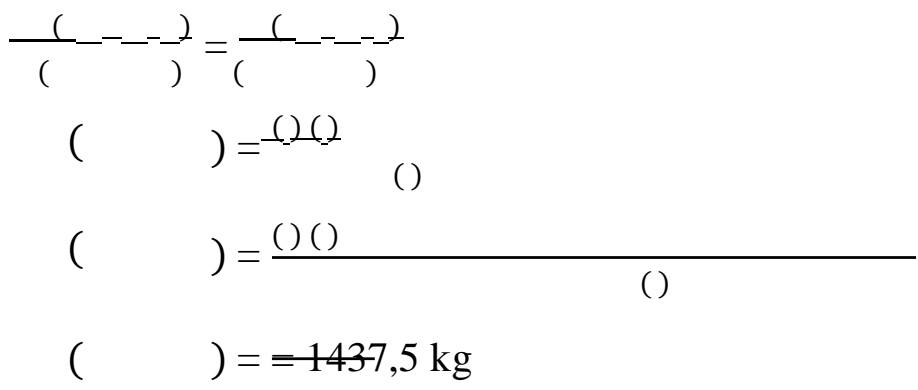

c) $\mathrm{m}($ ácido salicílico $)=185 \mathrm{~kg}$

$\mathrm{m}($ ácido acético $)=125 \mathrm{~kg}$

"Se trata de saber la cantidad de aspirina que se obtiene cuando reaccionan las sustancias"

$$
\begin{aligned}
& =\stackrel{()}{()}=1,48- \\
& =\stackrel{()}{()}=1,35-
\end{aligned}
$$

el reactivo limitante es el denominador:

Eso significa que el reactivo limitante es el anhídrido acético.

Toca ahora hallar la cantidad de aspirina que se obtiene o produce.

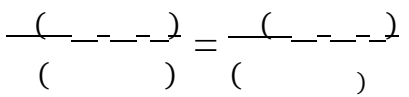

$$
\begin{aligned}
& (\quad)={ }_{(}^{()(---} \\
& (\quad)=()(2 \\
& =220,588 \mathrm{~kg}
\end{aligned}
$$


d) Rendimiento porcentual

$$
\begin{aligned}
& \text { X } 100= \\
& =82,2 \%
\end{aligned}
$$

a)

$$
\mathrm{x}=
$$

b).

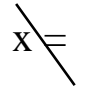

Esto quiere decir que si la eficiencia fuera del $100 \%$ se requeriría de pero solo el $80 \%$ de se convierte por tanto haremos la siguiente relación. 1 $\mathrm{kg}$ de reactivo del $80 \%$ $\mathrm{X}$ $0,8 \mathrm{~kg}$ de puro de $\mathrm{X}=1,44 \mathrm{x} \quad \mathrm{kg}$ de reactivo

O también: $=1,44 \mathrm{~kg}$ de reactivo

c). $185 \mathrm{~kg}$ ácido salicílico $\mathrm{x}$

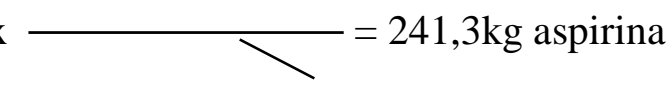

$125 \mathrm{~kg}$ anhídrido acético $\mathrm{x}$ $=220,5 \mathrm{~kg}$ aspirina

(Rendimiento teórico)

El reactivo que produce la menor cantidad de producto es el reactivo limitante o sea es el anhídrido acético.

e) Rendimiento porcentual $=-\times 100 \%=$

$$
-\mathrm{x} 100 \%=82,5 \%
$$

\section{Conclusiones.}

Con este solucionario sobre la aplicación de las leyes estequiometria a los cálculos químicos para sustancias en disolución, logramos: 
- Diferenciar y aplicar las unidades físicas y químicas más usadas para expresar la concentración de las disoluciones.

- Aplicar compresivamente expresiones matemáticas en la resolución de ejercicios relacionados con las disoluciones.

- Diferenciar entre rendimiento real y rendimiento teórico en una reacción química.

- $\quad$ Resolver ejercicios numéricos, empleando el concepto de reactantes limite.

- Calcular el reactante límite y el rendimiento de algunas reacciones químicas.

\section{Bibliografía.}

Álvaro M., F., (1998) Química, Escuela Politécnica del Litoral, Guayaquil.

Ander, P.y Sonnessa, A. J., (1992) Principios de Química, Editorial Limusa México.

Barbor, J. A. y Ibarz, J., (1979) Química General Moderna, Editorial Marín

Brady, J. E. y Humiston, G E., (1992) Química Básica, Editorial Limusa México.

Brow, T .L. - H.Euge Lsmay, Jr. - Química la Ciencia Centra, BruceE.

Bursten, Editorial Printice-Hall-Hispanoamericana S.A., Quito-Ecuador.

Bucheli Ponce, F., (1999) Química, Escuela Politécnica del Litoral, Guayaquil

Cárdenas, S. F., Gelvez C.A., (1996) Química y Ambiente 1, Editorial Mcgraw- Hill, Impreso en Colombia.

Chand, R., (1996) Química, Editorial Mcgraw- Hill Interamericana de Mexico.

Ebbing, D. D., (1997) Química General, Editorial Mcgraw - Hill Interamericana de Editores.

Escobar Castañeda, L.A., (1997) Fundamentos de Química en General, Escuela Politécnica del Ejercito, Quito.

Shaum, D. (1989) Química General Editorial Mcgraw - Hill impreso en Colombia. 